PROCEEDINGS OF THE

AMERICAN MATHEMATICAL SOCIETY

Volume 129, Number 6, Pages 1609-1615

S 0002-9939(00)05816-0

Article electronically published on October 31, 2000

\title{
ANTICHAINS OF MONOMIAL IDEALS ARE FINITE
}

\author{
DIANE MACLAGAN
}

(Communicated by Michael Stillman)

\begin{abstract}
The main result of this paper is that all antichains are finite in the poset of monomial ideals in a polynomial ring, ordered by inclusion. We present several corollaries of this result, both simpler proofs of results already in the literature and new results. One natural generalization to more abstract posets is shown to be false.
\end{abstract}

\section{Introduction}

Throughout this paper, $S=k\left[x_{1}, \ldots, x_{n}\right]$, where $k$ is a field. Our main result is the following theorem:

Theorem 1.1. Let $\mathcal{I}$ be an infinite collection of monomial ideals in a polynomial ring. Then there are two ideals $I, J \in \mathcal{I}$ with $I \subseteq J$.

If the monomial ideals were all principal, this would be Dickson's Lemma, or a special case of the Hilbert Basis theorem. The theorem is not true if the word "monomial" is omitted. A simple counterexample is the collection of ideals $\{\langle x-a\rangle$ : $a \in k\}$ where $S=k[x]$ and $k$ is a infinite field.

Although the statement of Theorem 1.1 may appear to be purely algebraic, monomial ideals are highly combinatorial objects. In particular, the above theorem can be restated as follows:

Theorem 1.2. Let $L$ be the poset of dual order ideals of the poset $\mathbb{N}^{n}$, ordered by containment. Then $L$ contains no infinite antichains.

A special case of interest is Young's lattice, which consists of the set of all partitions ordered by containment of Ferrers diagrams. Noting that a partition can be considered to be a finite order ideal in $\mathbb{N}^{2}$, we consider the generalized Young's lattice of finite order ideals in $\mathbb{N}^{n}$ ordered by inclusion.

Theorem 1.3. All antichains in the generalized Young's lattice are finite.

In the next section we give some corollaries of Theorem 1.1 Some of the corollaries have appeared in the literature before, but Theorem 1.1 allows us to simplify the original proofs, and provides a common framework for finiteness results involving monomial ideals. In Section 3 we give an application to SAGBI bases which was the motivating example for this paper. In Section 4 we outline an example which

Received by the editors September 15, 1999.

1991 Mathematics Subject Classification. Primary 13P10; Secondary 06A06, 52B20.

Key words and phrases. Posets, monomial ideal, Gröbner bases.

(C) 2000 American Mathematical Society 
shows that one natural generalization to more abstract posets is false, and lastly in Section 5 we give a proof of the theorem.

\section{Corollaries}

In this section we give several corollaries of Theorem 1.1 ,

The first corollary is a new proof of a basic result in computational algebra. A fundamental notion in Gröbner basis theory is that of an initial ideal of an ideal in a polynomial ring $S$. Given a term order $\prec$ (a total order on monomials in $S$ satisfying certain conditions), we define the initial term of a polynomial to be the largest monomial with respect to $\prec$ occurring in the polynomial. The initial ideal $i n_{\prec}(I)$ of $I$ with respect to $\prec$ is the monomial ideal generated by the initial terms of all polynomials in $I$. The following theorem appears in [2] and [5], and is well known.

Corollary 2.1. For a given ideal $I \in S$ there are only finitely many distinct initial ideals in $n_{\prec}(I)$.

Proof. The monomials of $S$ outside $i n_{\prec}(I)$ form a $k$-basis for $S / I$. If there were infinitely many initial ideals, then Theorem 1.1 would give a proper inclusion of $k$-bases.

Given an $\mathbb{N}^{d}$ grading on $S$, we can define the Hilbert series of a homogeneous ideal by

$$
H_{S / I}(t)=\sum_{b \in \mathbb{N}^{d}}\left(\operatorname{dim}_{k}(S / I)_{b}\right) t^{b}
$$

where $t^{b}=\prod_{i=1}^{d} t_{i}^{b_{i}}$.

Corollary 2.2. There are finitely many monomial ideals with a given Hilbert series with respect to a given grading.

Theorem 1.2 is also true when $\mathbb{N}^{n}$ is replaced by a finitely generated submonoid (such as the lattice points inside a rational cone).

Corollary 2.3. Let $M$ be a finitely generated submonoid of $\mathbb{N}^{n}$. Let $R=k[M]=$ $k\left[t^{a_{1}}, \ldots, t^{a_{d}}\right]$ be its monoid algebra. A monomial ideal in $R$ is an ideal generated by elements of the form $t^{b} \in R$ for some $b \in \mathbb{N}^{n}$. Then in any infinite collection $\mathcal{I}$ of monomial ideals in $R$ there are two, $I, J \in \mathcal{I}$, such that $I \subseteq J$.

Proof. Consider the map $\phi: k\left[x_{1}, \ldots, x_{d}\right] \rightarrow R$ given by $\phi: x_{i} \mapsto t^{a_{i}}$. For a monomial ideal $I \subseteq R$, we define $I_{\phi}=\left\langle x^{a}: \phi\left(x^{a}\right) \in I\right\rangle$. Then $I_{\phi} \subseteq J_{\phi} \Rightarrow I \subseteq J$, so the result follows from applying Theorem 1.1 to the set $\mathcal{I}_{\phi}=\left\{I_{\phi}: I \in \mathcal{I}\right\}$.

A similar corollary relates to $A$-graded algebras, where $A$ is a $d \times n$ matrix with entries in $\mathbb{N}$. An $A$-graded algebra is a $k$-algebra $R$ generated by $x_{1}, x_{2}, \ldots, x_{n}$ with an $\mathbb{N}^{d}$ grading (given by $\operatorname{deg} x_{i}=a_{i}$, where $a_{i}$ is the $i$ th column of $A$ ) such that $\operatorname{dim}_{k} R_{b}=1$ whenever $b \in \mathbb{N} A$ (the image of the map $\pi: \mathbb{N}^{n} \rightarrow \mathbb{N}^{d}$ given by $\pi: \omega \mapsto A \omega)$ and equals 0 otherwise. See [7, Chapter 10] for details of $A$-graded algebras.

Corollary 2.4. Let $R$ be an A-graded algebra. Let $\mathcal{I}$ be an infinite collection of ideals of $R$ which are homogeneous with respect to the A-grading. Then there are two ideals, $I, J \in \mathcal{I}$ such that $I \subseteq J$. 
Proof. $R$ is isomorphic to $S / I$ for some binomial ideal $I$. Any element of $S / I$ which is homogeneous with respect to the $\mathbb{N}^{d}$ grading can be written as $m+I$ where $m$ is some monomial in $S$, so homogeneous ideals of $R$ lift to monomial ideals in $S$. Containment in $S$ implies containment in $R$, so the result follows.

A trivial example of an $A$-graded algebra is $k\left[x_{1}, \ldots, x_{n}\right]$ with $A$ the $n \times n$ identity matrix. Then Corollary [2.4 reduces to Theorem 1.1.

\section{Applichtion to SAGBi bases}

Let $T=R\left[c_{1} x^{a_{1}}, \ldots, c_{n} x^{a_{n}}\right]$ be a monomial subalgebra of $R\left[x_{1}, \ldots, x_{d}\right]$, where $R$ is a Principal Ideal Domain. A strong SAGBI (Subalgebra Analogue to Gröbner Bases for Ideals) basis for $T$ is a collection $\left\{k_{1} x^{b_{1}}, \ldots, k_{m} x^{b_{m}}\right\}$ such that any element $c x^{l} \in T$ can be written as $c x^{l}=r \prod_{i=1}^{m}\left(k_{i} x^{b_{i}}\right)^{\phi_{i}}$ for some $\phi \in \mathbb{N}^{m}$ and $r \in R$.

Definition 3.1. Given a matrix $A \in \mathbb{N}^{d \times n}$, we define a map $\pi: \mathbb{N}^{n} \rightarrow \mathbb{N}^{d}$ by $\pi: y \mapsto A y$. Let $\mathbb{N} A \subseteq \mathbb{N}^{d}$ be the image of $\pi$. For $b \in \mathbb{N} A$ let $P_{b}=\operatorname{conv}\left(\pi^{-1}(b)\right)$. Since $\pi^{-1}(b)$ is a finite set, this is a convex polytope. We call $P_{b}$ the fiber of $A$ over $b$. A fiber over $b$ is atomic if there do not exist $b_{1}, b_{2} \in \mathbb{N}^{d}$ with $b_{1}+b_{2}=b$ such that $P_{b}=P_{b_{1}}+P_{b_{2}}$, where the addition is Minkowski sum.

Atomic fibers were defined by Adams et al. in [1], where they proved that there are only a finite number of atomic fibers for a given matrix $A$. They used this result to construct a finite strong SAGBI basis as follows:

Theorem 3.2 (Adams et al. [1]). Let $T=R\left[c_{1} x^{a_{1}}, \ldots, c_{n} x^{a_{n}}\right]$ and let $A=$ $\left[a_{1}, \ldots, a_{n}\right]$ be the $d \times n$ matrix with columns the $a_{i}$. Then $\left\{k_{b} x^{b}: P_{b}\right.$ is an atomic fiber of $A\}$ is a strong SAGBI basis for T, where $k_{b}=\operatorname{gcd}\left(\left\{c^{u}=c_{1}^{u_{1}} \ldots c_{l}^{u_{l}}: u=\right.\right.$ $\left.\left.\left(u_{1}, \ldots, u_{n}\right) \in \pi^{-1}(b)\right\}\right)$.

The proof of the finiteness result in [1] was constructive but complicated, using convex geometry techniques. Theorem 1.1 gives a much simpler, though nonconstructive, proof of this result.

Corollary 3.3. For a given matrix $A \in \mathbb{N}^{d \times n}$, there are only a finite number of atomic fibers.

Proof. For $b \in \mathbb{N} A$, let $I_{b}=\left\langle x^{u}: A u=b\right.$ and $u$ is a vertex of $\left.P_{b}\right\rangle$. Then the fiber over $b$ is atomic if and only if $I_{b}$ is not contained in any $I_{b^{\prime}}$ for $b \neq b^{\prime}$. If there were an infinite number of atomic fibers, then $\left\{I_{b}: P_{b}\right.$ atomic $\}$ would be an infinite antichain of monomial ideals, contradicting Theorem 1.1 .

Corollary 3.3 can be generalized as follows:

Definition 3.4. Let $M$ be a monomial ideal of $S$, and $A \in \mathbb{N}^{d \times n}$ a matrix. Then the $(M, A)$ fiber over $b \in \mathbb{N} A$ is the set $\left\{u: A u=b\right.$ and $\left.x^{u} \notin M\right\}$. An $(M, A)$ fiber over $b$ is atomic if there do not exist $b_{1}, b_{2} \in \mathbb{N} A$ with $b_{1}+b_{2}=b$ such that for all $u$ in the $(M, A)$ fiber over $b$ there are $u_{1}, u_{2}$ in the $(M, A)$ fibers over $b_{1}, b_{2}$ respectively such that $u=u_{1}+u_{2}$.

To see that this definition is a generalization of an earlier one, we first need another definition. 
Definition 3.5. Given a matrix $A \in \mathbb{N}^{d \times n}$, we define its vertex ideal, $V_{A}$, by

$$
V_{A}=\bigcap_{\prec} i n_{\prec}\left(I_{A}\right)
$$

where the intersection is over all term orders $\prec$, and $I_{A}$ is the toric ideal corresponding to $A$ (see [7] for details on toric ideals).

Note that this is a finite intersection by Corollary 2.1 Since the standard monomial of $A$-degree $b$ of an initial ideal of a toric ideal corresponds to a vertex of $P_{b}$, and each vertex of $P_{b}$ is standard for some initial ideal, the set of standard monomials of $V_{A}$ is exactly $\left\{x^{u}: u\right.$ is a vertex of $\left.P_{A u}\right\}$. Thus Definition 3.1 is Definition 3.5 with $M=V_{A}$.

Corollary 3.6. There are only finitely many atomic $(M, A)$ fibers for given $M$ and $A$.

The proof is the same as for Corollary [3.3. Of particular interest is the case $M=(0)$. In that case, being atomic corresponds to the nonexistence of $b_{1}, b_{2}$ such that each lattice point in $\pi^{-1}(b)$ is a sum of lattice points in $\pi^{-1}\left(b_{1}\right)$ and $\pi^{-1}\left(b_{2}\right)$, as opposed to the original definition, where only the vertices need be sums of lattice points in the two smaller fibers. This is a strictly stronger requirement. The following example shows that a fiber can be atomic with respect to this stronger definition without being atomic in the original sense.

Example 3.7. Let $A$ be the following matrix:

$$
\left(\begin{array}{llllll}
1 & 1 & 1 & 0 & 0 & 0 \\
0 & 3 & 2 & 1 & 0 & 0 \\
5 & 0 & 2 & 0 & 1 & 0 \\
0 & 2 & 1 & 0 & 0 & 1
\end{array}\right) .
$$

Let

$$
b_{1}=(1,3,5,2)^{T} \quad \text { and } \quad b_{2}=(5,10,10,6)^{T} .
$$

We have

$$
\begin{aligned}
\pi^{-1}\left(b_{1}\right)=\{ & (1,0,0,3,0,2)^{T}, \\
& (0,1,0,0,5,0)^{T}, \\
& \left.(0,0,1,1,3,1)^{T}\right\}
\end{aligned}
$$

and

$$
\begin{aligned}
\pi^{-1}\left(b_{2}\right)=\{ & (0,0,5,0,0,1)^{T}, \\
& (1,2,2,0,1,0)^{T}, \\
& \left.(2,3,0,1,0,0)^{T}\right\} .
\end{aligned}
$$

Now $P_{b_{1}+b_{2}}=P_{b_{1}}+P_{b_{2}}$, so $b_{1}+b_{2}$ is not atomic in the first sense. However $(1,1,4,2,2,2)^{T} \in \pi^{-1}\left(b_{1}+b_{2}\right)$, but cannot be written as the sum of lattice points in $P_{b_{1}}$ and $P_{b_{2}}$. This example is based on an example of Oda $[6]$ for lattice polytopes. 


\section{Poset formulation}

From the second formulation of the theorem, it is natural to suspect that this is in fact a general theorem about posets. Two properties of the poset $\mathbb{N}^{n}$ which lend themselves to finiteness results are that $\mathbb{N}^{n}$ has no infinite antichains, and that it satisfies the descending chain condition. Such posets are known as wellquasi-ordered. The following example consists of a poset which has no infinite antichains or infinite descending chains such that the poset of dual order ideals under containment contain an infinite antichain. This example appears in [3], but was discovered independently by George Bergman, from whom I learned it.

Example 4.1 (G. Bergman [3]). Let $X$ be the set $\{(i, j): i, j \in \mathbb{N}, i<j\}$. Set $(i, j) \prec\left(i^{\prime}, j^{\prime}\right)$ if and only if $j<j^{\prime}$ and either $i=i^{\prime}$ or $j<i^{\prime}$. It is straightforward to check that $X$ is a partially ordered set.

Note that any chain descending from $(i, j)$ can have at most $j-1$ members less than $(i, j)$, so there are no infinite descending chains of elements of $X$. To see that all antichains in $X$ are finite, suppose $Y$ is an antichain in $X$, and let $j_{0}$ be the smallest $j$ such that $(i, j) \in Y$, occurring in the pair $\left(i_{0}, j_{0}\right) \in Y$. Then $(i, j) \in Y$ implies $i \leq j_{0}$, as otherwise $j_{0}<j$, and then $\left(i_{0}, j_{0}\right) \prec(i, j)$. If there are two pairs $(i, j),\left(i, j^{\prime}\right) \in Y$, with $j<j^{\prime}$, then $(i, j) \prec\left(i, j^{\prime}\right)$, so there is only one pair of the form $(i, j) \in Y$ for each value of $i$. But this means there are at most $j_{0}+1$ elements in $Y$, so all antichains in $X$ are finite.

Because there are no infinite descending chains or infinite antichains each dual order ideal in $X$ can be represented by its finite antichain of minimal elements. One dual order ideal is contained in another exactly when each element of the finite antichain of minimal elements of the first dual order ideal is greater than some element of the finite antichain of minimal elements of the second.

For fixed $l>0$, let $S_{l}=\{(k, l): k<l\} \subseteq X$. Then $S_{l}$ is the finite antichain of minimal elements of a dual order ideal of $X$. Suppose the dual order ideal determined by $S_{l_{2}}$ is contained in the one determined by $S_{l_{1}}$. From above, we must have $l_{1}<l_{2}$. But then there is no element of $S_{l_{1}}$ less than $\left(l_{1}, l_{2}\right) \in S_{l_{2}}$, a contradiction. So the $S_{l}$ form an infinite antichain of dual order ideals of $X$.

Theorem 1.2 can, however, be generalized in the following way:

Theorem 4.2 (Farley, Schmidt [4]). Let $P$ and $Q$ be two posets with no infinite antichains that satisfy the descending chain condition. If the posets of dual order ideals of $P$ and of $Q$, ordered by inclusion, have no infinite antichains, then the same is true for the poset of dual order ideals of $P \times Q$.

\section{Proof of the MAIN THEOREM}

In this section we give the proof of Theorem 1.1 .

We first prove Theorem 1.3. The generalized Young's lattice is isomorphic to the poset of artinian monomial ideals under inclusion, via the map taking an order ideal to its complement, so we prove the theorem in that setting.

Lemma 5.1. Let $\mathcal{I}$ be an infinite collection of artinian monomial ideals (primary to the maximal ideal). Then there are two ideals, $I, J \in \mathcal{I}$ such that $I \subseteq J$.

Proof. Suppose $\mathcal{I}$ consists of an infinite number of artinian monomial ideals, which are noncomparable with respect to inclusion. Choose $I_{1} \in \mathcal{I}$. Since $I \nsubseteq I_{1}$ for $I \in \mathcal{I} \backslash\left\{I_{1}\right\}$, each $I \in \mathcal{I} \backslash\left\{I_{1}\right\}$ contains some of the finite number of standard 
monomials of $I_{1}$. There are thus an infinite number of ideals in $\mathcal{I}$ which contain the same set of standard monomials of $I_{1}$. Call this collection $\mathcal{I}_{1}$. Let $J_{1}$ be the intersection of the ideals in $\mathcal{I}_{1}$. We will now build a strictly ascending chain of monomial ideals. Suppose $\mathcal{I}_{k}$ and $J_{k}$ have been chosen. Choose an ideal $I_{k+1} \in \mathcal{I}_{k}$. We can again find an infinite collection of ideals in $\mathcal{I}_{k}$ which have the same nontrivial intersection with the standard monomials of $I_{k+1}$. Let $\mathcal{I}_{k+1}$ be this collection, and let $J_{k+1}$ be the intersection of the ideals in $\mathcal{I}_{k+1}$. We have $J_{k+1} \supsetneq J_{k}$, since $J_{k+1}$ contains some standard monomials of $I_{k+1}$, so in this fashion we get an infinite ascending chain of monomial ideals in $S$, which is impossible.

Corollary 5.2. Let $\mathcal{I}$ be an infinite collection of artinian monomial ideals. Then there is an infinite chain $I_{1} \supsetneq I_{2} \supsetneq \ldots$ of ideals in $\mathcal{I}$.

Proof. Since $S$ is Noetherian, $\mathcal{I}$ contains maximal ideals. There are only finitely many maximal ideals by Lemma 5.1, so set $I_{1}$ to be a maximal ideal in $\mathcal{I}$ containing an infinite number of ideals of $\mathcal{I}$, and repeat, setting $\mathcal{I}=\left\{I \in \mathcal{I}: I \subsetneq I_{1}\right\}$.

Proof of Theorem 1.1. Every associated prime of a monomial ideal is a monomial prime, of which there are only a finite number. We can thus restrict to an infinite collection of $\mathcal{I}$ all of which have the same set of associated primes, which we will also call $\mathcal{I}$. Now for each ideal in this set we find an irredundant primary decomposition, writing the ideal as the intersection of monomial ideals primary to an associated prime in such a way that each associated prime is used only once. Let $I_{\tau}$ be the primary component of $I$ primary to the monomial prime $P_{\tau}=\left\langle x_{i}: i \notin \tau\right\rangle$, where $\tau \subseteq[n]$. For a fixed $\tau$ either $\left\{I_{\tau}: I \in \mathcal{I}\right\}$ is finite, so there is an infinite number of $I \in \mathcal{I}$ with the same $I_{\tau}$, or we can apply Corollary 5.2 to the polynomial ring $k\left[x_{i}: i \notin \tau\right]$. In either case we get an infinite collection $\mathcal{I}_{\tau}=\left\{I_{k}: k \geq 1\right\}$ of ideals in $\mathcal{I}$ such that $I_{1 \tau} \supseteq I_{2 \tau} \supseteq \ldots$, where the inclusions need not be proper. Since there are only a finite number of associated primes, by appropriate restrictions we can find a sequence $\left\{I_{k}: k \geq 1\right\}$ such that $I_{1} \supseteq I_{2 \tau} \supseteq \ldots$ for each $\tau$ such that $P_{\tau}$ is an associated prime. But since $I_{k}$ is the intersection of the $I_{k \tau}$, where $\tau$ ranges over all associated primes $P_{\tau}$ of $I_{k}$, this means that $I_{1} \supsetneq I_{2} \supsetneq \ldots$, where the inclusions are proper, since the $I_{k}$ are all distinct.

\section{ACKNOWLEDGMENTS}

The proof of Theorem 1.1 was much improved from a previous version by discussion with Dave Bayer.

\section{REFERENCES}

[1] William W. Adams, Serkan Hoşten, Philippe Loustaunau, and J. Lyn Miller. SAGBI and SAGBI-Gröbner bases over principal ideal domains. J. Symbolic Comput., 27:31-47, 1999. MR 99j:13023

[2] David Bayer and Ian Morrison. Standard bases and geometric invariant theory. I. Initial ideals and state polytopes. J. Symbolic Comput., 6(2-3):209-217, 1988. Computational aspects of commutative algebra. MR 90e:13001

[3] D. Duffus, M. Pouzet, and I. Rival. Complete ordered sets with no infinite antichains. Discrete Math., 35:39-52, 1981. MR 82j:06003

[4] Jonathan D. Farley, 1998. Private communication.

[5] Teo Mora and Lorenzo Robbiano. The Gröbner fan of an ideal. J. Symbolic Comput., 6(23):183-208, 1988. Computational aspects of commutative algebra. MR 90d:13004 
[6] Tadao Oda. Problems on Minkowski sums of convex lattice polytopes. Preprint. 7 pages.

[7] Bernd Sturmfels. Gröbner Bases and Convex Polytopes. American Mathematical Society, Providence, RI, 1996. MR 97b:13034

Department of Mathematics, University of California, Berkeley, California 94720 E-mail address: maclagan@math.berkeley.edu

Current address: School of Mathematics, Institute for Advanced Study, Einstein Drive, Princeton, New Jersey 08540

E-mail address: maclagan@ias.edu 